\title{
Una rappresentazione digitale del castello Eurialo per l'indagine storico-interpretativa
}

\author{
A Digital representation of Euryalus fortress: a historical interpretive study
}

\author{
Rita Valenti ${ }^{\text {a }}$, Sebastiano Giuliano ${ }^{\text {b }}$, Emanuela Paternò ${ }^{c}$ \\ ${ }^{a}$ Dipartimento di Ingegneria Civile e Architettura, Università degli Studi di Catania, Syracuse, Italy, r.valenti@unict.it \\ ${ }^{b}$ Facoltà di Ingegneria e Architettura, Università degli Studi di Enna Kore, Enna, Italy, sebastiano.giuliano@unikore.it \\ ${ }^{c}$ Dipartimento di Ingegneria Civile e Architettura, Università degli Studi di Catania, Syracuse, patermanu@ hotmail.it
}

\begin{abstract}
It is clear that concepts and cognitive processes aimed at putting forward fortified systems in their relation with the territory and with the surrounding landscape, establish inextricably interwoven "interests" and a consequent osmotic hysteresis between their emergence and disappearance into the deepest part of the earth. The logic behind the defensive structures of Euryalus fortress is particularly interesting. The fortress designed during the Greek period and located on the top of Epipolae hill, represented a strategic fortification for the city's defence. Therefore, it is reasonable to think about a sequence of emerging or disappearing empty spaces; a semantic vacuum to be filled with logical-subjective interpretations pertaining to those who visit the place. The conceptual framework provides knowledge and documentation meant as indispensable supporting instruments to understand Dionysius I and Archimedes' thoughts where the integration of people and environment forms the basis of the close iterative connection among nature, artifice and landscape. The research takes this direction implementing innovative technological systems trying to go beyond in order to achieve virtual reconstruction processes, even if partial, of the fortress. Survey, thus, can be applied as an instrument for the knowledge of historical heritage which once converted into digital heritage is a support for the reconstruction of lost ancient scenarios. Actually, the implementation of innovative systems allows an easy-to-use data viewing which supports the interpretive phase, the archiving, consultation and dissemination of survey products. In particular, the survey of the fortress with integrated methodologies (both instrumental and photogrammetric) provides not only a fundamental basis for the documentation of the fortress conditions but also provides a basis for the collection of reconstructive hypotheses formulated by researchers who have been involved with the structure so far. Interactions among archaeology, history, geomorphology and technology make history and a past renowned glory come alive with the common spirit of coming up to a strong synergy between the past and the future destiny of the place.
\end{abstract}

Keywords: Cultural heritage, Euryalus fortress, survey, historical analysis

\section{Tecnologie digitali e patrimonio culturale}

La tutela del patrimonio culturale, che rappresenta uno degli obiettivi e al tempo stesso una delle sfide di maggiore importanza dei giorni nostri, si realizza altresì con la creazione di si- stemi innovativi per la conservazione e la fruizione della conoscenza. Conoscere vuol dire documentare ed "il documentare" costituisce il primo step per la salvaguardia del patrimonio 
culturale. In tal senso sono state sviluppate moderne tecnologie di acquisizione ed elaborazione dei dati digitali attraverso le quali è possibile documentare e archiviare il patrimonio storicoarchitettonico e divulgarne la memoria storica. Nello specifico, il processo conoscitivo dell'Architettura si concretizza nella generazione di prodotti metrici ottenuti grazie all'impiego di metodologie differenti di rilevo (metodi imagebased e range-based) più o meno sofisticate. I dati ricavati dalle campagne di rilievo rappresentano uno strumento di conoscenza e costituiscono un supporto per la creazione di prodotti multimediali destinati al grande pubblico. In particolare, tridimensionalità e virtualità rappresentano, oggigiorno, gli elementi principali per la comunicazione dei Beni Culturali. La rappresentazione digitale, infatti, si sta gradualmente affermando come strumento efficace per la comprensione e la comunicazione del patrimonio costruito. Di fatto, la documentazione grafica costituisce un momento essenziale dell'indagine soprattutto nel caso in cui l'interpretazione, la conservazione e la valorizzazione riguarda il patrimonio archeologico. È proprio il caso del dominante e possente castello Eurialo (Fig. 1).

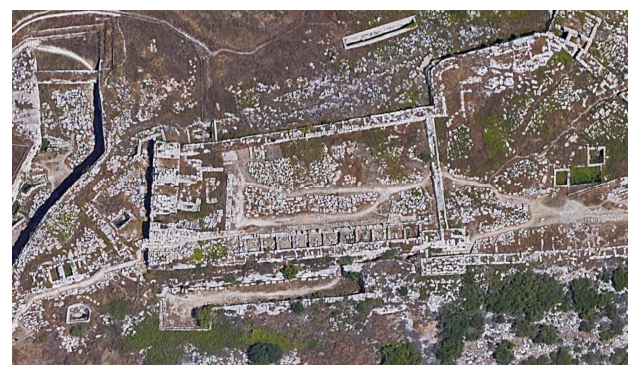

Fig. 1. Castello Eurialo: veduta aerea dei resti.

Forma, geometria, materia, consistenze murarie e misura di manufatti, sommati all'insieme di tutte le documentazioni di supporto, costituiscono la base di una banca dati utile per l'articolazione dei processi conoscitivi della fabbrica e degli intendimenti profondi, sottesi dall'acuto profilo geniale di Dionisio il Vecchio e di Archimede, tutti rivolti all'interesse verso la città di Siracusa, per la difesa dalle incursioni nemiche. La frattalità di tutto ciò che circonda a livello ambientale il castello Eurialo costituisce l'interesse e la materia prima delle nostre indagini e dei nostri obiettivi culturali (Fig. 2). Nel caso specifico, le tecnologie digitali messe in atto offrono una descrizione formale e metrica accurata del territorio e dei reperti, offrendo uno strumento efficace per l'analisi e la visualizzazione del sito. È opportuno operare tenendo conto, sia nelle fasi di acquisizione che in quelle di post-processamento dei dati digitali, delle caratteristiche di ogni tecnica e strumento adoperato al fine di ottenere un prodotto valido per l'archiviazione e la condivisione. In una visione più ampia, nel rivisitare i luoghi del castello Eurialo, sarebbe auspicabile creare una rete di laboratori di monitoraggio e di controllo del territorio che, dislocati in maniera diffusa, riescano a realizzare e determinare, come in un processo osmotico, un continuo trasferimento di informazioni, indispensabili per la documentazione del patrimonio storico delle fortificazioni e delle loro implicazioni sul territorio. Tali laboratori, mutuamente collegati ed interagenti, sono equiparabili ai cosiddetti Osservatori Ambientali, capaci di incamerare dati, di monitorare e controllare, con un ampio raggio di azione, tutto l'insieme sistemico del documentare. Il nostro compito non può essere solo e semplicemente limitato allo studio sempre più approfondito del castello, della Torre, del Maniero, della Fortificazione ma, anzi, deve affrontare, con forza di intenti e precipuamente, tematiche che lascino i segni tangibili di un impegno rivolto al tramandare ai posteri i criteri e le logiche strutturanti degli assetti difensivi presi in esame. Dunque, il lavoro di ricerca proposto è rivolto a creare, a partire da una campagna di rilievi condotta con la recente tecnologia TLS, messa a disposizione dal Laboratorio della Rappresentazione della SDS di Siracusa dell'Università degli Studi di Catania, una mappatura del sito per individuarne lo stato di conservazione e una documentazione metrica e grafica utile per effettuare eventuali approfondimenti culturali e individuare modalità di rappresentazione tridimensionale opportune per la comprensione e la diffusione. 


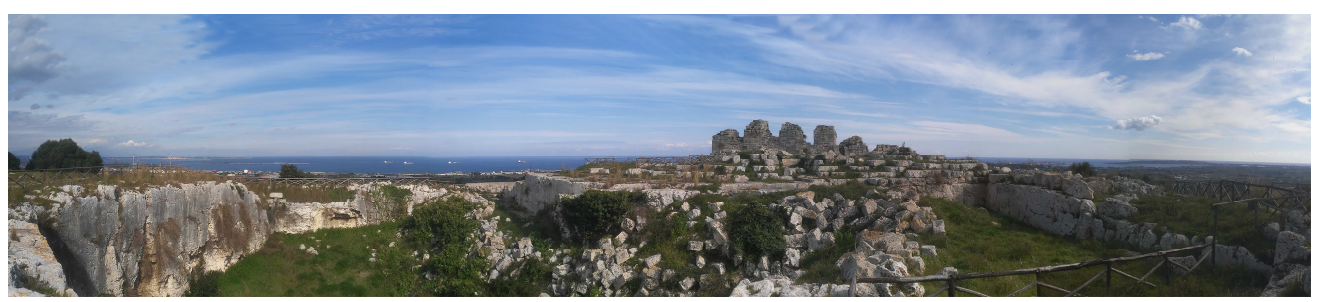

Fig. 2. Vista panoramica dai resti del castello Eurialo.

\section{Passi nel castello Eurialo tra immagini sto- riche e modelli visuali}

"Il luogo racconta storie sedimentarie che ne testimoniano i significati originari e le vicissitudini che lo hanno trasformato nel corso del tempo. [...] La rovina è object trouvé, acquista valori estetici e di uso museografico che oltrepassano il suo proposito originario" (Pinto, 2008, p. 4). Questa oggi la straordinaria fortezza dell'Eurialo, posta al vertice occidentale in corrispondenza della zona più elevata della terrazza dell'Epipoli, importante testimonianza del sistema difensivo lungo $27 \mathrm{~km}$, voluto dal tiranno Dionigi e costruito tra il 402 e il 397 a.C. (Voza, 1994, pp. 209-210). Da questo luogo con la vista è possibile controllare tutto il territorio intorno: Ortigia con i suoi due porti, tutta la costa sino al golfo di Catania. Il sito è citato da Tucidide come «un continuo pendio fino alla Città, e da questo si osserva tutto; e dai Siracusani è chiamato Epipole la punta perché di molto sovrasta al restante" (Tucidide, libro VI, p. 96). Proprio a causa della sua vulnerabilità, sperimentata nella guerra contro Atene, Dionigi provvide in tempi rapidissimi, così come racconta Diodoro Siculo, alla realizzazione dell'opera di fortificazione costituita da una cinta muraria che circondava tutto l'Epipoli fino al mare.

"Certamente punto di maggiore interesse di questo immenso sistema difensivo è rappresentato dalla fortezza nota come "castello Eurialo", [...] dalla particolare forma a punta cui pare alluda il nome greco Euryelos (testa di chiodo) che passò a denominare la vicina fortezza" (Voza, 1994, pp. 211-212).

In particolare, un'immagine del sistema fortificato, seppure fantastica, la si ritrova nella famosa incisione di Vincenzo Mirabella del 1613; tale rappresentazione esprime le conoscenze del tempo sulle antichità greche ricavate, come lo stesso Mirabella riporta nel proemio, dalla lettura di Tucidide, Diodoro, Plutarco, Livio, Cicerone, Virgilio, Pomponio Mela e Strabone, e nel caso della fortificazione dell'Epipoli da indagini sui luoghi ${ }^{1}$. Questa rappresentazione ebbe molta fortuna tra i viaggiatori del grand tour. Hoüel la propone nel suo Voyage pittoresque des isles de Sicile, de Malte et de Lipari (Fig. 3) ribadendone i contenuti.

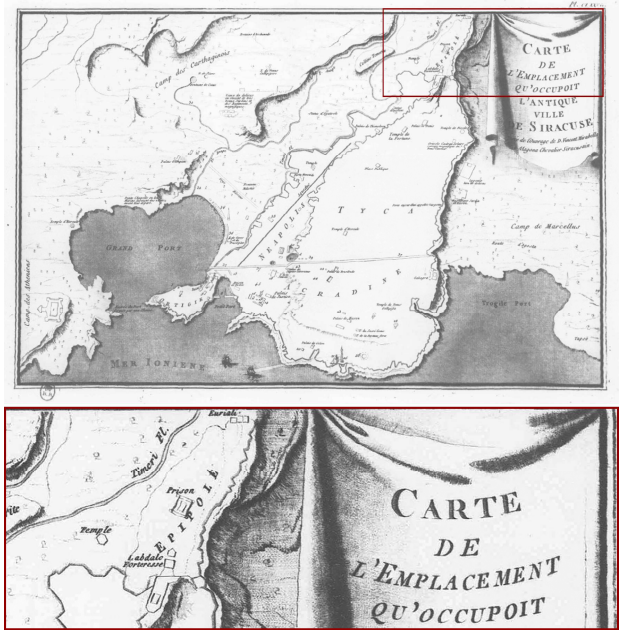

Fig. 3. In alto, J. Hoüel 1782, vol III (1785), tav: CLXXVII: Carte de l'emplacement qu'occupait l'antique ville de Siracuse, Tirée de l'ouvrage de D.Vincent Mirabella et Alagona Chevalier Siracusain. Incisione 26x38 cm; in basso, dettaglio (Beneventano del Bosco, 1995, p. 105).

La complessità morfologica dei ruderi, attribuiti in base a "criteri scientifici" al castello Eurialo nella seconda metà del XIX secolo ${ }^{2}$, è stata riportata alla luce dopo una intensa e altrettanto complessa campagna "di scavi mirata alla ricostruzione planimetrica delle possenti opere di difesa" (Zirone, 2011, p. 186). In realtà l'area, do- 
po l'interesse a riscoprire il mondo antico da parte degli studiosi del cinquecento e del seicento (Arezzo, Fazello, Mirabella, Cluverio, Bonanni), è stata, nell'ottocento, oggetto di studi e scavi da parte di F.S. Cavallari e Holm, all'inizio del novecento da parte di P. Orsi e dei generali Rocchi e Schramm fino allo studio del Mauceri del 1928 e alle indagini di G.V. Gentili tra il 1959 e il 1961 (Zirone D., 2011, in Ampolo 2011, pp.186-189; Voza 1994, p. 212) (Fig. 4).

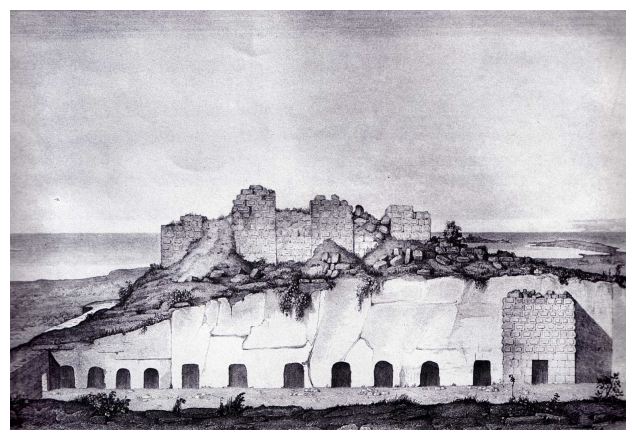

Fig. 4. Elevazione degli avanzi del castello Eurialo. Di Giovanni da un disegno di F. S. Cavallaro, in D. Lo Faso, duca di Serradifalco, Le Antichità della Sicilia, Palermo 1834-40. Incisione 28,5 x $42 \mathrm{~cm}$ (da Beneventano del Bosco 1995, 177).

Oggi, l'obiettivo è di continuare la ricerca e verificare come le tecnologie attuali e le nuove metodologie della visualizzazione possano contribuire a fornire supporti più avanzati per gli studi in atto. Le potenzialità della conservazione e documentazione di frammenti di porzioni di manufatti architettonici stratificati si interfaccia con l'esigenza di ritrovare e trasmettere anche i riferimenti materici non più esistenti, per fornire una conoscenza completa e complessa, nella fattispecie, di un paesaggio antropo-geografico che, nonostante i secoli intercorsi e le mutazioni al contorno non sempre in sintonia con i luoghi, proprio per le sue caratteristiche naturali ha mantenuto quelle peculiarità che sostanzialmente ne hanno determinato, circa 2500 anni fa, l'uso e la conformazione antropica. L'utilizzo di software dedicati alla rappresentazione consente una visualizzazione dei dati del rilievo che agevola nella fase interpretativa, nell' archiviazione e nella consultazione e diffusione dei prodotti del rilievo (Fig. 5).

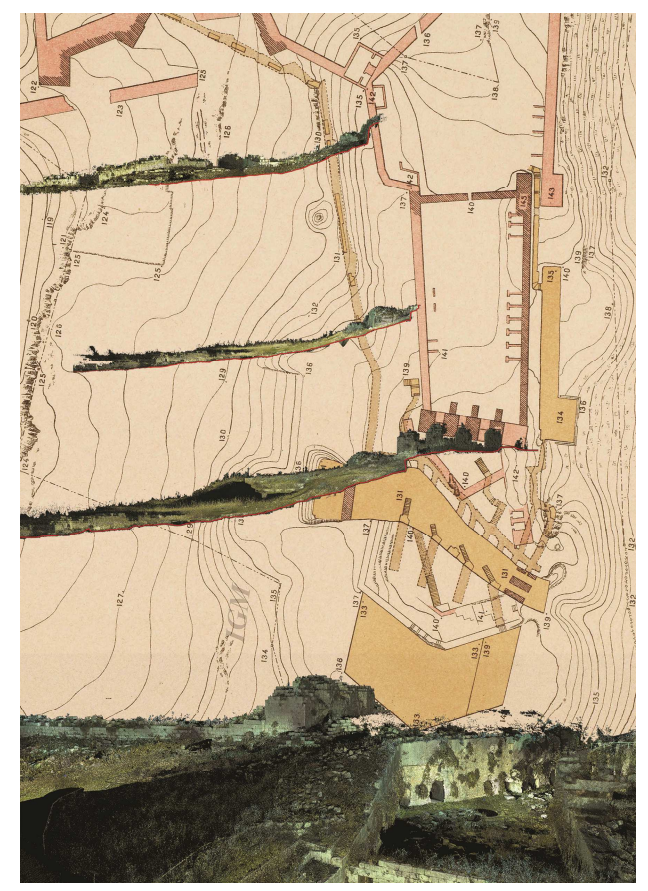

Fig. 5. Visualizzazione del rilievo a nuvola di punti dell'area indagata. Carta base: Rilievo tacheometrico eseguito dal topografo principale Alessandrini, marzo 1913. Stampa a colori 66 x $50 \mathrm{~cm}$ (da Istituto Geografico Militare, cartografia F. 646 (Siracusa Sez. II), dettaglio.

Questa risulta particolarmente utile, soprattutto, quando la materia con cui ci si confronta è costituita da frammenti rotti e corrosi a causa della vetustà e dell'abbandono per secoli. Lo studio condotto approda alla elaborazione, scientificamente e intellettualmente rigorosa, di un modello digitale che assume il ruolo di documento visuale, fondamentale per la interpretazione dei reperti archeologici e per la divulgazione della conoscenza. Tale modello si conforma a partire dal rilievo digitale dei luoghi e della materia esistente secondo un sistema di misura mediante "sensori attivi" dei ruderi ${ }^{3}$. In questa ottica il processo di elaborazione della visualizzazione attraverso il computer delle ipotesi formulate si compie mediante la chiara e immediata esplicitazione delle fasi metodologiche di lettura, in modo che siano sempre riconoscibili sia la parte oggettiva del lavoro di restituzione sia la parte interpretativa con i relativi riferimenti di supporto. 


\section{I modelli congetturali: tra validazione e ri- costruzione}

La ricostruzione congetturale del castello Eurialo necessita di un'adeguata conoscenza dell'ambito studiato in relazione alle vicende storiche e militari cui ne hanno caratterizzato l'intera fabbrica difensiva. Sono i contributi dei più saperi che nel loro stratificarsi rendono possibili rappresentazioni grafiche, oggi anche immersive-virtuali, di opere simbolo ed icona del territorio, come quello siracusano, con il suo castello Eurialo. Sono diversi gli studi che secondo un excursus storico-archeologico, a scopo umanistico e scientifico, cercano di dare una visione d'insieme, nel tentativo di comprendere al meglio una delle più complesse opere di ingegneria militare dell'antichità Siracusana, riconosciuta nel 2005 Patrimonio dell'Umanità. Diversi sono gli studiosi che già da inizio ottocento ne hanno esaminato i resti ed illustrato i primi risultati delle proprie ricerche. Tra questi particolarmente significativi appaiono gli studi di Domenico Lo Faso Pietrasanta duca di Serradifalco, di Francesco Saverio Cavallari e di Luigi Mauceri, che ne elabora un' ampia trattazione sia sugli aspetti storico-militari, sia sugli aspetti architettonici, finalizzati ad una ricostruzione congetturale in scala uno duecento dell'intera fabbrica. Secondo gli studi tutta la costruzione presenta diversi elementi strategici, appositamente progettati per cogliere di sorpresa gli eventuali assalitori:

"[...] Nel castello Eurialo si trovano largamente applicati i principi dell'arte difensiva di tutti i tempi. Le opere avanzate, costituite da tre fossati, disposti trasversalmente alla dorsale, ed il duplice recinto del corpo principale, dimostrano che il concetto della resistenza successiva o scalare, del quale si fece un'applicazione eccessiva dagli ingegneri militari del secolo XVII, era ben noto nell'antichità. In quelle disposizioni che la tecnica costruttoria, assai progredita degli architetti militari greci riuscì a rendere perfettamente corrispondenti allo scopo, mercé il copioso sviluppo delle comunicazioni sotterranee, si rivela al più alto grado il concetto della difesa attiva. Il principio della massa inerte e della difesa passiva, il quale, rappresentato dalle gigantesche muraglie, potrebbe credersi la caratteristica dell'architettura militare dell'antichità, cede il posto, nella fortezza siracusana, alla mobilità, alla elasticità ed alla attività della resistenza [...]" (Rocchi, 1908, pp. 41-42).

Sono i disegni di Francesco Saverio Cavallari del 1880 (Fig. 6) a dare una prima restituzione grafica bidimensionale dell' antica fortezza. I disegni non tralasciano l'aspetto territoriale su cui il castello è incastonato.

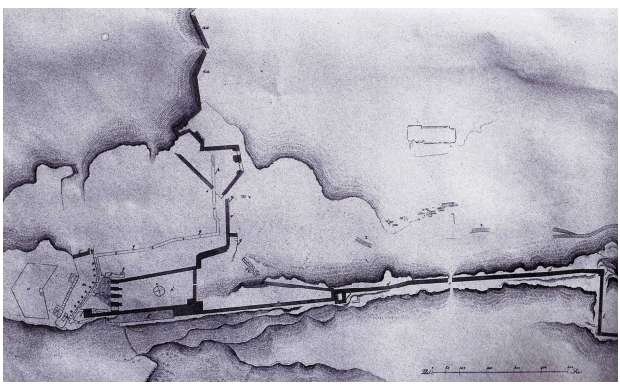

Fig. 6: Pianta degli avanzi del castello Eurialo. F.S. Cavallaro, in D. Lo Faso, duca di Serradifalco, Le Antichità della Sicilia, Palermo 1834-40. Incisione 28,5 x $42 \mathrm{~cm}$ (da Beneventano del Bosco 1995, 176).

Sono rappresentazione su più scale di approfondimento, che vanno dai disegni di rilievo della scala territoriale con le tracce della muraglia, alla ricostruzione planimetrica del mastio ${ }^{4}$ e dei tre fossati del castello ${ }^{5}$, alle ricostruzioni di dettaglio del profilo longitudinale all'estremità occidentale della terrazza siracusana. Anche la ricostruzione congetturale di Luigi Mauceri si sviluppa a partire dalle informazioni bidimensionali sugli aspetti topografici rilevati ed ipotizzati dal Cavallari. L'apporto del Mauceri consiste essenzialmente nello sviluppo tridimensionale degli alzati del mastio, delle torri e degli ambienti che si sviluppavano attorno i fossati. Nelle rappresentazioni prospettiche, ricostruite secondo un dettaglio di scala uno duecento, non vengono tralasciati gli aspetti territoriali del luogo, facendo emergere il rapporto tra la fortezza ed il paesaggio Siracusano. Secondo una ricostruzione di dettaglio, invece, il prospetto delle cinque torri viene ipotizzato con una struttura lignea di tamponamento che si innesta tra gli speroni della torre.

Sempre secondo una ricostruzione minuziosa, il Maugeri non si ferma alla ricostruzione conget- 
turale della zona del mastio e dei fossati: nella sua opera sono presenti dettagliati disegni di ipotesi sulle torri della muraglia. Sono tutte ricostruzioni validate da un approfondimento storiografico sui sistemi di difesa di epoca antica, in cui il rilievo delle macerie pervenute diviene la base su cui ipotizzare gli alzati in funzione delle consuetudini costruttive storico-architettoniche e dall'arte della difesa militare. Gli attuali mezzi di rilievo digitale permettono di generare modelli tridimensionali a nuvole di punti da cui è possibile sviluppare precisi elaborati grafici bidimensionali e tridimensionali, ad elevato gradiente informativo, riguardo gli aspetti materici e geometrici dei resti del castello (Fig. 7).

In relazione alle possibili ricostruzioni virtuali della fortezza, il primo, del duplice obiettivo della ricerca è quello di porre il modello a nuvola di punti, derivante dal rilievo digitale, come base metrica e morfologica di dettaglio da cui sviluppare la modellazione solida tridimensionale. In tal modo, sulla base del modello solido 3D, è possibile integrare tutte le possibili ricostruzioni congetturali delle parti distrutte dell'opera architettonica. In tal senso, se il rilievo laser scanner offre la precisa base geometrica della struttura, la ricerca storiografica sui metodi costruttivi e sulle strategie di difesa della fortezza danno la possibilità di completare il quadro conoscitivo del castello, con lo scopo di restituire una lettura globale dell'oggetto, dove ogni singola parte viene raccordata all'intero sistema difensivo.

Il secondo obiettivo, invece, è quello di porre il modello a nuvola di punti come base metrica e morfologica su cui validare tutte le ricostruzioni congetturali della fortezza, al fine di stabilire precise corrispondenze ed evidenziare eventuali difformità tra i modelli ricostruiti e i dati acquisiti tramite le ormai consolidate tecnologie di rilevamento digitale. Lo scopo è quello di arrivare a formulare un indice delle ricostruzioni congetturi, validate sulla base scientificamente accreditata del rilievo digitale, al fine di stabilirne parti congruenti e parti difformi all'attuale conformazione architettonica dei resti della fortezza.

In tal senso, sulla base dei risultati del metodo d'indagine è possibile arrivare a formulare tutta una serie di possibili ricostruzioni congetturali, da modellare virtualmente grazie gli attuali mezzi di rappresentazione tridimensionale e rendere fruibili tramite e le possibilità offerte dai più innovativi mezzi di fruizione virtuale. Il rilievo laser scanner dell'opera, oltre a fornire una importante base documentaria sullo stato della fortezza, pone le basi per raccogliere tutte le ipotesi ricostruttive che gli studiosi, nel corso degli anni e supportati da indagini storiografiche, hanno sviluppato ed impresso con china su rappresentazioni cartacee di indubbio valore (Fig. 8).

Gli attuali mezzi di rappresentazione virtuale, oltre a validare e rendere fruibili tridimensionalmente le ricostruzioni prodotte in passato, possono innescare importanti meccanismi di fruizione virtuale del luogo e delle sue possibili ricostruzioni, innescando processi che tendono alla valorizzazione culturale del bene, con notevoli ricadute che spaziano dal piano economico al piano della conoscenza.

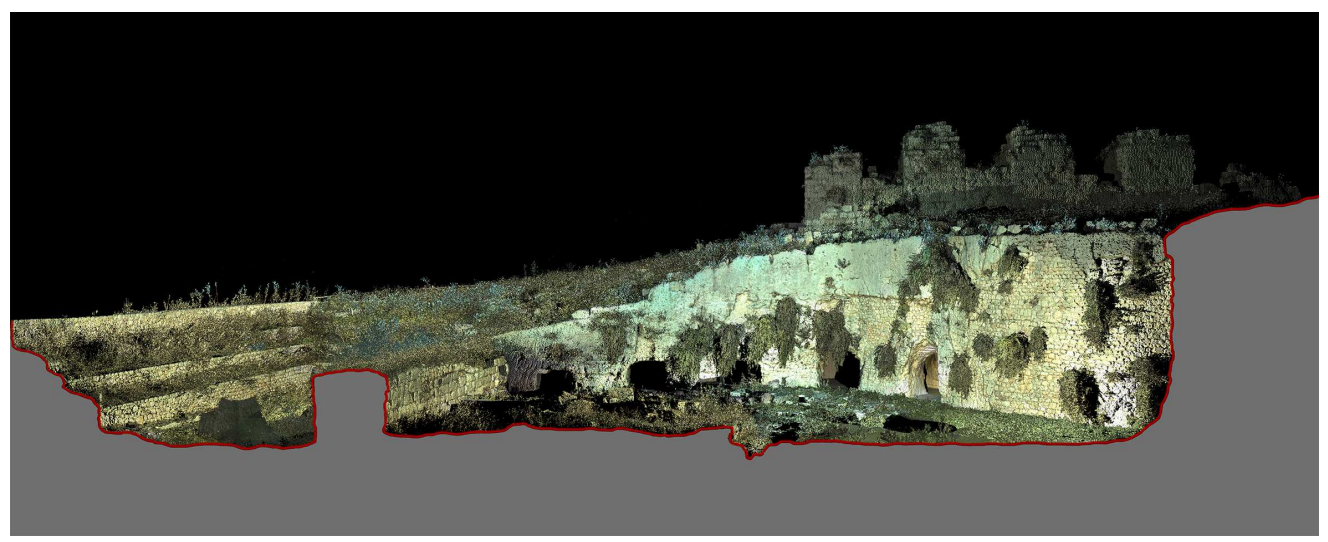

Fig. 7: Rilievo laser scanner, sezione trasversale del secondo fossato. 

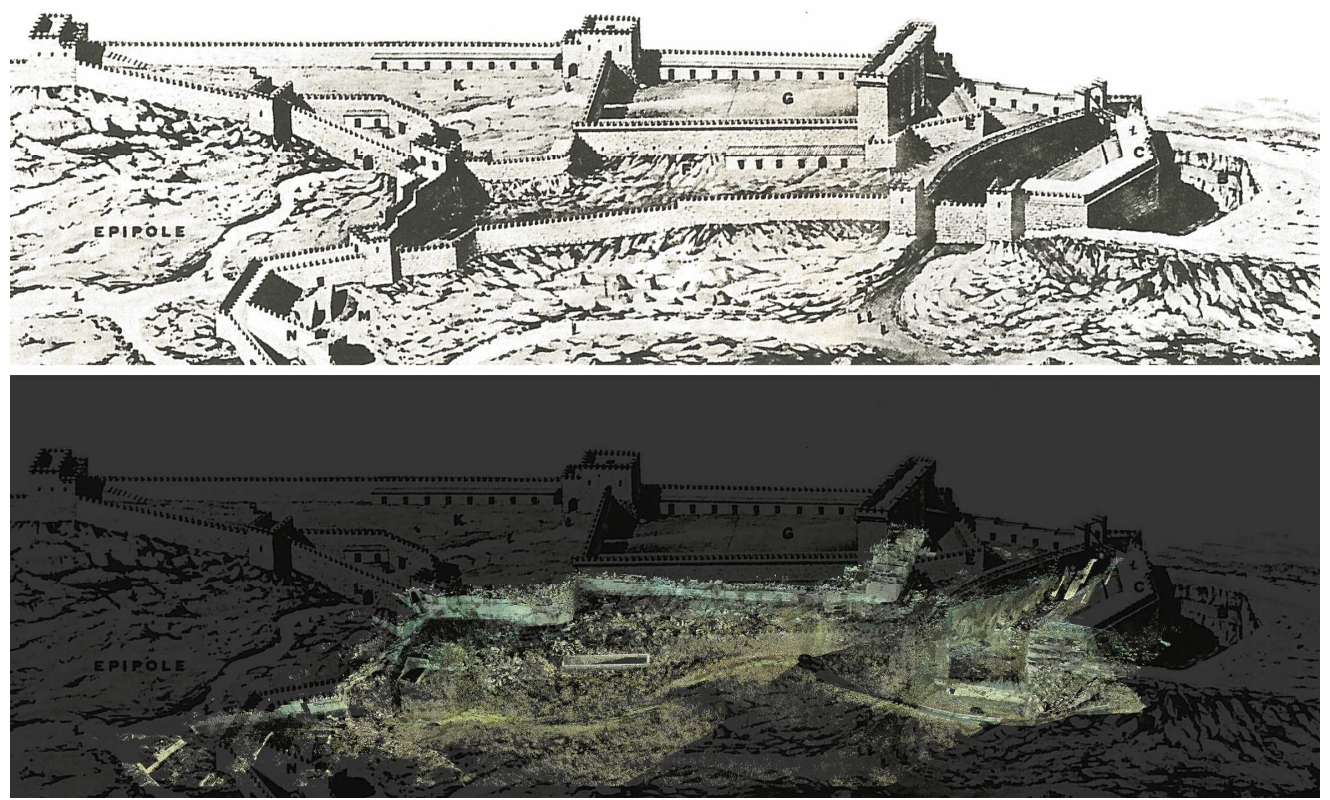

Fig. 8. Tra rilievo e validazione: modello a nuvola di punti e ricostruzione congetturale di Luigi Mauceri (Mauceri, $1939, \operatorname{tav} 1)$.

\section{Conclusioni}

L'obiettivo del lavoro di ricerca non si limita alla ricostruzione-interpretazione dei dati scaturenti dalla ricerca documentaria delle fonti attuali e dei dati acquisiti sul campo secondo i dettami dei principi della Carta di Londra sulla virtual archaeology, ma vuole restituire la contestualizzazione paesaggistica del topos, fondamentale per comprendere la storia del sistema artefattonatura espressione di una dialettica dinamica e complessa tra manufatto architettonico stratificato e paesaggio geografico. L'incontro tra archeologia, storia, geomorfologia e tecnologia contribuisce a mantenere viva una storia e una gloria appartenenti ad un passato noto a tutti, nell'intento di creare una forte sinergia tra passato e destino futuro di questo luogo.

\section{Note}

Lo studio è il risultato della collaborazione di tutti gli autori. Nello specifico, Rita Valenti ha scritto il paragrafo 2 e le conclusioni; Emanuela Paternò ha scritto il paragrafo 1; Sebastiano Giuliano ha scritto il paragrafo3.
${ }^{1}$ Salvatore Russo, scheda de L'Antiche Siracuse di D. Vincenzo Mirabella e Alagona dedicate alla S.C.R.M. del re FilippoIII Nostro Signore, in Beneventano del Bosco (1995), pp. 41-49.

${ }^{2}$ Cfr. Zirone D. 2011, in Ampolo 2011, pp.185186. Per l'attribuzione dei ruderi al castello è di interesse il racconto del Mauceri $(1928,1939)$ nel capitolo IV sulla "Topografia del Castello e la sua denominazione".

${ }^{3}$ I rilievi sono stati effettuati con la strumentazione in dotazione del Laboratorio della Rappresentazione della Struttura Didattica Speciale di Architettura della Università degli Studi di Catania, con sede in Siracusa.

${ }^{4}$ A forma trapezoidale, recuperato in epoca bizantina elevando i relativi spazi interni. I bizantini, inoltre, a chiusura del mastio edificarono cinque torri su pianta quadrata, coronate da grondaie a testa leonica.

${ }^{5}$ L'entrata del castello è protetta da tre fossati più uno laterale. Il primo, più piccolo, è lungo 6 $\mathrm{m}$ e profondo 4 , mentre il secondo, a $86 \mathrm{~m}$ dal primo, è lungo circa $50 \mathrm{~m}$ a difesa di un'opera 
avanzata alle spalle della quale si trova il terzo fossato largo $17 \mathrm{~m}$ e profondo $9 \mathrm{~m}$ che collega il sistema difensivo con dei sotterranei. Nella parte sud del terzo fossato tutte le ricostruzioni raffigurano un ponte levatoio che consentiva il superamento del fossato tra i due gruppi di fortificazioni. Esso era anche raggiungibile attraverso una scalinata interna che dal fossato ne permette l'accesso. Infine sul versante sud era presente un quarto fossato che difendeva il punto meno ripido della parete laterale del pendio dell'Epipoli. Questo fossato è collegato al terzo tramite accessi sotterranei.

\section{Bibliography}

Ampolo, C., ed. (2011). Siracusa. Immagine e storia di una città, Edizioni della Normale Pisa.

Beneventano del Bosco barone di Monteclimiti, P., ed. (1995). Siracusa urbs magnificentissima. La collezione Beneventano di Monteclimiti, Electa Milano.

Ciancio, S. (1980). Siracusa e provincia. Topografia storica e archeologica, Edizioni Dafni Catania.

Gringeri Pantano, F., ed. (2003). Jean Hoüel. Voyage a Siracusa. Le antichità della città e del suo territorio nel 1777. Catalogo della mostra (Siracusa, 8 maggio-8 giugno 2003), Sellerio Palermo.

Mauceri, L. (1939). Il castello Eurialo nella storia e nell'arte, Ristampa della II edizione (1993), Edizioni Dafni Catania.

Pagello, E. (2004). "Siracusa", in Magnano di San Lio, E.; Pagello, E., eds., Difese da difendere. Atlante delle Città Murate di Sicilia e Malta, Officine Grafiche Riunite Palermo, Fondazione Culturale "Salvatore Sciascia" Caltanissetta.

Pinto, J.C. (2008). "Il progetto sincronico", Rivista di Architettura del Dipartimento di Architettura Rappresentazione e Progetto, 1, Edizioni della Meridiana, Firenze, pp. 4-19.

Rocchi, E. (1908). Le fonti storiche dell'architettura militare, Officina Poligrafica Editrice Roma.

Voza, C. (1994). Guida di Siracusa, ERRE Produzioni Siracusa. 